complications (bleeding/pain) were analysed. Pearson chisquare tests were used to compare experiences by gender, high vs. low levels of socioeconomic deprivation (using Index of Multiple Deprivation scores), and whether patients reported receiving sedation or not.

Results After excluding patients outside the target date range and those who did not have colonoscopy, 76,717 patients were eligible for analysis, of whom 60,581 (79.0\%) responded to the questionnaire. Nearly all patients felt they understood the risks (95.7\%) and benefits (98.2\%) of the test, and 97.8\% felt the preparation instructions were clear. Comparison by gender and deprivation did not yield clinically meaningful $(\geq 3 \%)$ differences. In terms of the hospital experience, virtually all patients felt they were treated with respect $(98.5 \%)$ and had privacy $(98.0 \%)$, but $20.8 \%$ experienced more discomfort than expected (although only 5.2\% asked for the test to be stopped/paused). Procedural discomfort was moderated by gender, with more women than men reporting higher-thanexpected discomfort $(25.4 \%$ vs. $17.9 \%$; p $<0.0005)$, and requesting that the test be stopped/paused $(7.1 \%$ vs. $3.9 \%$; $<0.0005)$. Use of sedation showed only a weak association with patient experience: $22.2 \%$ of sedated vs. $20.2 \%$ of nonsedated patients reported unexpected discomfort; $6.4 \%$ vs. $4.8 \%$ asked for the test to be stopped/paused; both p-values $<0.0005$ ). Post-test, $14.3 \%$ of patients reported pain and $6.9 \%$ reported rectal bleeding. Pain was more common in women $(18.0 \%$ vs. $11.9 \%$; $<0.0005)$ but there were no other clinically meaningful differences post-test related to gender or deprivation level.

Conclusion Most patients referred for colonoscopy as part of the Bowel Cancer Screening Programme have a positive colonoscopy experience. The most negative aspect of the experience was the test being unexpectedly uncomfortable. Patients are extensively counselled pre-procedure but more emphasis on managing expectations, along with continued measures to reduce discomfort and pain are required, particularly for women.

Disclosure of Interest None Declared.

\section{PWE-036 USE OF AN ULTRASLIM PAEDIATRIC COLONOSCOPE TO COMPLETE DIFFICULT COLONOSCOPIC PROCEDURES}

D Tate*, P Dunckley. Gloucestershire Royal Hospital, Gloucester, UK

\subsection{6/gutjnl-2014-307263.296}

Introduction Discomfort and failure to progress beyond the sigmoid are the commonest reasons for non-completion of colonoscopy ${ }^{1}$ We anecdotally observed that the use of a new ultraslim paediatric colonoscope (Olympus PCF-PQ260L) when the standard colonoscope failed often led to completion of difficult cases.

Methods We analysed 19 reports from cases at the Gloucestershire Royal hospital where the PCF-PQ260L was used as a second endoscope on an examination started with a regular colonoscope (Olympus H260 or Q260 colonoscopes) over the period January to August 2013.

Results 95\% (18/19) of the time the extent of a non-completed colonoscopy was the sigmoid colon. $50 \%$ of the time this was due to diverticular disease with patient discomfort the second most common cause. In $80 \%$ of these cases, the subsequent use of the PCF-PQ260L enabled the endoscopist to reach the caecum. In addition, in those procedures that failed due to discomfort, comfort scores were improved in $50 \%$ during the second procedure with the PCF-PQ260L. The
CIR of the GI consultants working at GRH averaged 96\% for 2013.

Conclusion The PCF-PQ260L enabled the negotiation of the sigmoid colon in $80 \%$ of cases where a standard endoscope failed in the hands of skilled colonoscopists. This limited study suggests that the PCF-PQ260L is an exciting new tool in the colonoscopist's inventory.

\section{REFERENCE}

1 Dafnis $G$, Granath $F$, Påhlman L, Ekbom A, Blomqvist P. Patient factors influencing the completion rate in colonoscopy. Dig Liver Dis. $2005 ; 37(2): 113-8$

Disclosure of Interest None Declared.

\section{PWE-037 PANENTERIC CAPSULE ENDOSCOPY: AN ALTERNATIVE NON-INVASIVE TOOL TO SCREEN FOR IDIOPATHIC INFLAMMATORY BOWEL DISEASE (IBD)}

E Ejenavi ${ }^{*}, M$ McAlindon, D Majumdar, R Sidhu, A Lobo, K Drew, S Hardcastle. Gastroenterology, Sheffield Teaching Hospitals NHS Foundation Trust, Sheffield, UK

\subsection{6/gutjnl-2014-307263.297}

Introduction Compared to conventional endoscopy, capsule endoscopy (CE) is potentially safer, non-invasive, performed in out-patients and may be an alternative first line investigation in patients with suspected inflammatory bowel disease (IBD). In colon CE (CCE), a dormant mode (to save battery) is followed by device activation when small bowel mucosa is recognised. In this pilot study patients with suspected small and/or large bowel disease underwent a pan-enteric assessment using combined small bowel (SBCE) and CCE.

Methods Patients underwent combined SCE and CCE using a novel protocol. Patients had new GI symptoms (group A: symptoms alone or those with additional abnormal results - GI symptoms plus) or underwent assessment of known IBD (group B). Main outcome measures: diagnostic yield (relevant findings only), complications, CE completion rates and colon cleanliness (scored 1-4: excellent to poor).

Results Patients (group A, $\mathrm{n}=56$; group $\mathrm{B}, \mathrm{n}=26$; mean age 41 ) had refused (50\%), had incomplete (21\%) prior colonoscopy or chose to have CCE (29\%). Group A patients had diarrhoea (62\%) and abdominal pain (54\%); 17 had GI symptoms plus anaemia (13), acute phase response (9), hypoalbuminaemia (4), radiological abnormalities (3). Mean SBCE and CCE SB examination times: 255 and 92 mins respectively. Mean C examination time: $167 \mathrm{mins}$; median cleanliness score 2. SBCE was complete in $73(89 \%)$ and CCE in 58 patients (71\%). In group $\mathrm{B}$, pathology was identified in 62\%, 16/26 (all active Crohn's) which was significantly higher than in Group A (20\%: 11/56, p $=0.0003)$. New diagnoses in Group A: Crohn's disease $(\mathrm{n}=5)$ and one each of NSAID colitis, proctitis, leiomyoma, angioectasia, diverticulae and idiopathic ulcerated small bowel stricture. 9/ 11 were in the symptoms plus group. 95\% of pathology identified on SBCE was also identified on CCE. No complications were reported.

Conclusion $62 \%$ of patients known to have IBD had active disease, but diagnostic yield was as high as $20 \%$ in those with new symptoms. IBD was the commonest and no complications occurred. Studies of the respective roles of faecal biomarkers, $\mathrm{CE}$ and histology in the diagnosis of IBD are needed. Almost all small bowel pathology was recognised by CCE suggesting its use as a remote panenteric endoscopic tool only awaits further battery development.

Disclosure of Interest None Declared. 